\title{
Interaction of chemical patterns in coupled layers
}

\author{
David G. Míguez, ${ }^{1,{ }^{*}}$ Milos Dolnik, ${ }^{2}$ Irving Epstein, ${ }^{2}$ and Alberto P. Muñuzuri ${ }^{3, *}$ \\ ${ }^{1}$ Department of Physics of Condensed Matter, Facultad de Ciencias, Universidad Autónoma de Madrid, Madrid 28049, Spain \\ ${ }^{2}$ Department of Chemistry, MS015, Brandeis University, Waltham, MA 02454-9110, USA \\ ${ }^{3}$ Group of Nonlinear Physics, Universidade de Santiago de Compostela, Campus Sur, Santiago de Compostela, Spain
}

(Received 17 December 2010; published 24 October 2011)

\begin{abstract}
We investigate the interaction between reaction-diffusion systems coupled by diffusion. The photosensitive CDIMA (chorine dioxide-iodine-malonic acid) reaction allows us to study experimentally the mutual influence of two layers of Turing patterns coupled via a diffusive interaction. By illuminating each of the layers with different intensities of homogeneous external light, the chemical conditions in each layer can be shifted, allowing us to study the result of diffusive interaction between Turing patterns with different spatial configurations. Our experiments suggest a complex scenario for the interaction between different patterns, strongly dependent on the spatial characteristics of the interacting patterns. Numerical simulations are also reported in full agreement with experimental observations.
\end{abstract}

DOI: 10.1103/PhysRevE.84.046210

PACS number(s): 05.45.-a, 82.40.Ck

\section{INTRODUCTION}

The study of coupling and synchronization is one of the most important and developed areas in nonlinear dynamics [1-3], strongly motivated by both biological and theoretical interests. From neural network models to chemical oscillators or chaotic synchronization, the way complex systems respond when coupled is often difficult to anticipate, even after extensive characterization of their behavior in an isolated environment $[4,5]$.

Interest in reaction-diffusion systems composed of spatially interacting layers has increased over the past years [6-9]. Nonlinear pattern formation systems have been successfully coupled numerically and experimentally in a variety of systems, such as the well-known CDIMA (chorine dioxideiodine-malonic acid) reaction $[10,11]$. This chemical system has the ability to exhibit Turing patterns $[12,13]$ due to a diffusion-driven instability. The well-characterized photosensitivity of the CDIMA reaction also has the advantage of being sensitive to light $[14,15]$. Its photosensitivity has been used to force transitions in the system in many different contexts [16-19]. In principle, homogeneous constant external intensity of illumination induces changes in the spatial configuration of the pattern, from a hexagonal configuration to a labyrinth pattern of stripes and a honeycomb-like arrangement of spots [20]. When the illumination is sufficiently high, the pattern can be also suppressed, leaving the system in a homogeneous steady state.

Here, we present experiments and numerical simulations performed in a Turing system composed of two coupled layers interacting via diffusion. We will take advantage of the photosensitive characteristics of the CDIMA reaction to study the interaction between different Turing pattern configurations by differentially illuminating the two layers. Our experimental setup allows us to induce a different spatial arrangement in each of the two-dimensional layers and analyze the interactions between spots, stripes, and honeycomb configurations. Our

\footnotetext{
*davidgmiguez@gmail.com, uscfmapm@cesga.es http://openwetware.org/wiki/User: DavidMiguez
}

results suggest that the outcome of the interaction is highly dependent on the spatial characteristics of the two patterns. We show, experimentally and numerically, how the two Turing structures rearrange spatially, inducing pattern intercalation (spots-spots and spots-stripes) and even pattern annihilation (spots-honeycomb).

Previous results by Berenstein et al. [8] have shown that the interaction between coupled layers in a Turing system determines the configuration of the final pattern, although the experimental setup used did not allow direct control of the configuration and observation of both patterns (patterns were obtained using different chemical reactants in each layer and observation was possible indirectly using optical filters to distinguish the structures). The experimental setup presented here allows us to illuminate each one of the interacting layers with a different intensity of homogeneous illumination to induce different chemical conditions on both layers, without using different chemical reactants. This setup allows us to perform a visual analysis of both layers to quantitatively describe the different transitions observed. Section II describes the experimental setup and procedures. Numerical model and simulation protocols are explained in Sec. III. In Sec. IV, results are described with the different transitions reported, and a complete phase diagram of the system. Finally, a discussion of the results is presented in Sec. V.

\section{EXPERIMENTAL SETUP}

Experiments were performed using the CDIMA reaction in a thermostated one-sided continuously fed unstirred chemical reactor (CFUR) coupled to a continuously fed stirred tank reactor (CSTR), where reagents are mixed. A scheme of the experimental setup and the chemical reactor used can be observed in Fig. 1; it is equivalent to other configurations previously used in the literature [21,22].

The chemical reactor was designed in such a way that allows observation of the pattern from both front and rear sides of the reactor. The main feature of this reactor is the ability to illuminate both sides with different light intensities, thus controlling the chemical concentrations in the system on both sides. The reactor was placed vertically and two digital cameras were used 

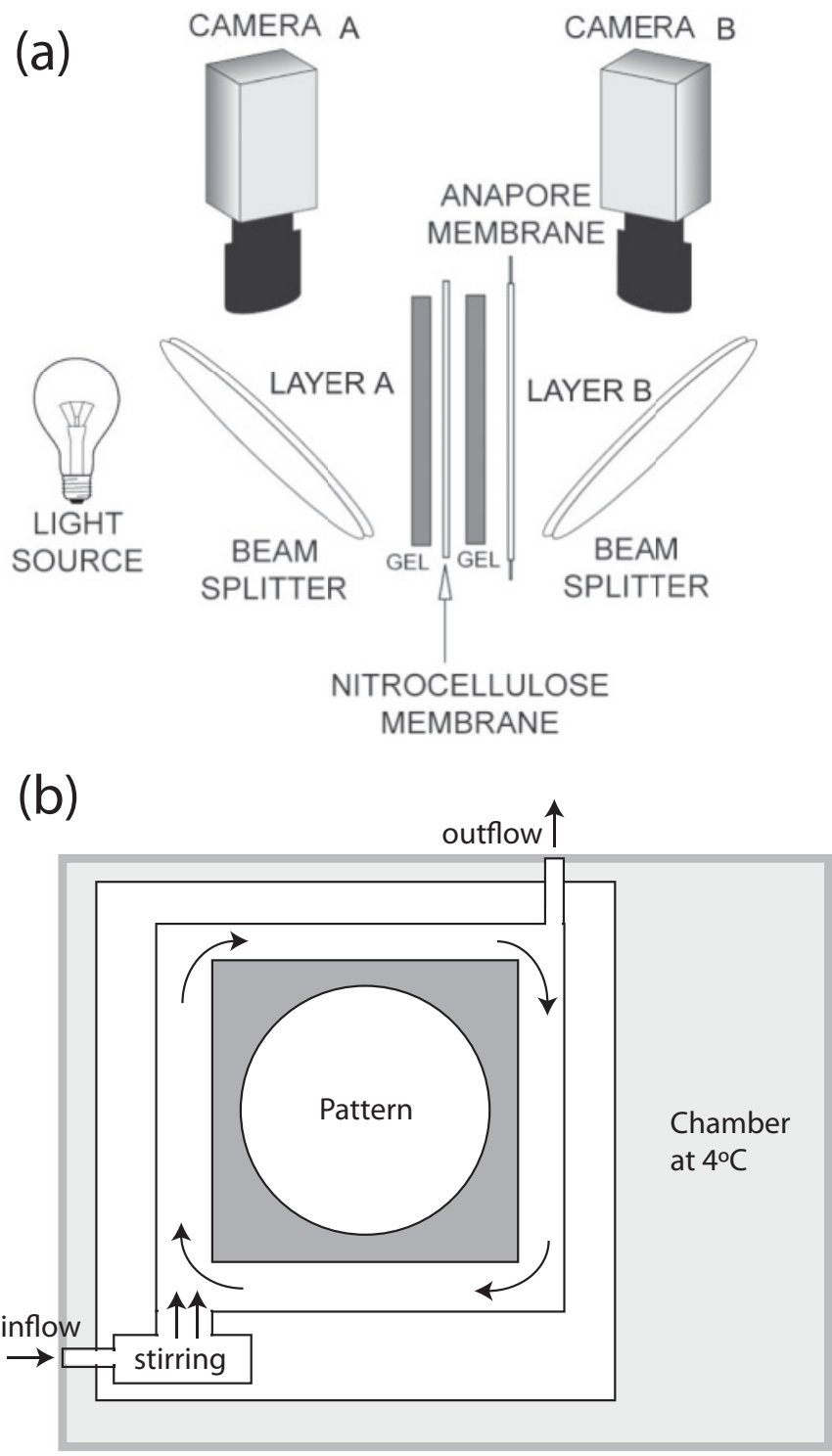

FIG. 1. (a) Scheme of the experimental setup used to perform the experiments. Light is focused onto one of the gels through a beam splitter, which allows the recording of the evolution of both patterns with two CCD cameras. (b) Transversal view of the vertical chemical reactor used for the experiments. The design of the reactor allowed observation of the pattern through the front and back sides. Chemical reactants flowed following the arrows and reached the center, where the CFURs (gels and membranes) were placed.

to monitor the resulting pattern from both sides. We maintained strong stirring conditions in the CSTR and low residence time to ensure constant chemical conditions throughout the experiment. To study the interaction of patterns coupled by diffusion, we placed inside the reactor two "two-dimensional" agarose gels (0.3-mm thickness each, $2 \%$ agarose), separated by an opaque nitrocellulose membrane (Schleicher and Schell, pore size $0.45 \mu \mathrm{m}$ ). The nitrocellulose membrane provides a white background that allows us to distinguish the pattern in both gel layers, while allowing differential illumination of the layers. This way, taking advantage of the photosensitiveness of the CDIMA reaction, the local chemical conditions in one of the layers can be shifted, while maintaining the chemical conditions of the other layer almost unchanged. The external light was used to induce a different pattern in both sides, and the nitrocellulose membrane sets the strength of the coupling by diffusion. Light illumination was provided by a video projector connected to a computer and focused onto the selected gel for the illumination.

Finally, an additional anapore membrane (Whatman, pore size $0.2 \mu \mathrm{m}$ ) was introduced to provide a rigid support of the two-gel system, as well as to avoid convection effects, acting as a physical separation between the CFUR and the CSTR. This anapore membrane was impregnated with a $2 \%$ agarose solution. This membrane is almost transparent when wet, so it does not prevent the visualization and illumination of the pattern.

The chemical conditions were fixed for all of the experiments: $\left[\mathrm{I}_{2}\right]_{0}=0.45 \mathrm{mM},\left[\mathrm{ClO}_{2}\right]_{0}=0.1 \mathrm{mM}$, [malonic acid $]=0.9 \mathrm{mM}, \quad[\text { poly }(\text { vinyl alcohol })]_{0}=10 \mathrm{~g} / \mathrm{L}$, and $\left[\mathrm{H}_{2} \mathrm{SO}_{4}\right]_{0}=10 \mathrm{mM}$; temperature $=4 \pm 0.5{ }^{0} \mathrm{C}$, residence time $500 \mathrm{~s}$. Under these conditions, and without differential illumination in the layers, the pattern develops in a similar way in both layers. The structure is composed mostly of spots arranged in a hexagonal configuration.

Since we used a one-side-fed chemical reactor, fresh reactant has to travel through one of the layers to reach the other. This configuration introduces a potential difference between the two gel systems inside the reactor (one of the layers is inevitably closer to the feeding chamber, so it will receive more fresh reactants than the other). This did not represent an issue for the high values of the residence time used here, and the two patterns show the same configuration when illuminated with the same light intensity [Figs. 2(a) and 2(b)].

A typical experiment is performed as follows: we leave the two-layer system to spontaneously develop patterns freely, which typically takes around 24 hours until they reach a stationary condition. We then proceed by illuminating one of the layers (which will be referred to as "layer $A$ " in the following) with constant and homogeneous light intensity, while no illumination is applied in the other "layer $B$." After applying the light, we allow the system to reach steady state again (around 3 hours), and then we take snapshots of the patterns in both illuminated and nonilluminated layers for and superposed (after specular transformation of layer $A$ ) for comparison.

\section{NUMERICAL SIMULATIONS}

In addition, to corroborate the experimental observations, two-dimensional numerical simulations were performed using the Lengyel-Epstein model for the CDIMA reaction, modified to take into account the light sensitiveness [14] and the dual layer configuration of the experimental system:

$$
\begin{aligned}
& \frac{\partial u_{a}}{\partial t}=a-c u_{a}-\frac{4 u_{a} v_{a}}{1+u_{a}^{2}}-\phi_{a}+\nabla^{2} u_{a}+k_{u}\left(u_{b}-u_{a}\right), \\
& \frac{\partial v_{a}}{\partial t}=\sigma\left(c u_{a}-\frac{u_{a} v_{a}}{1+u_{a}^{2}}+\phi_{a}+d \nabla^{2} v_{a}+k_{v}\left(v_{b}-v_{a}\right)\right) ;
\end{aligned}
$$




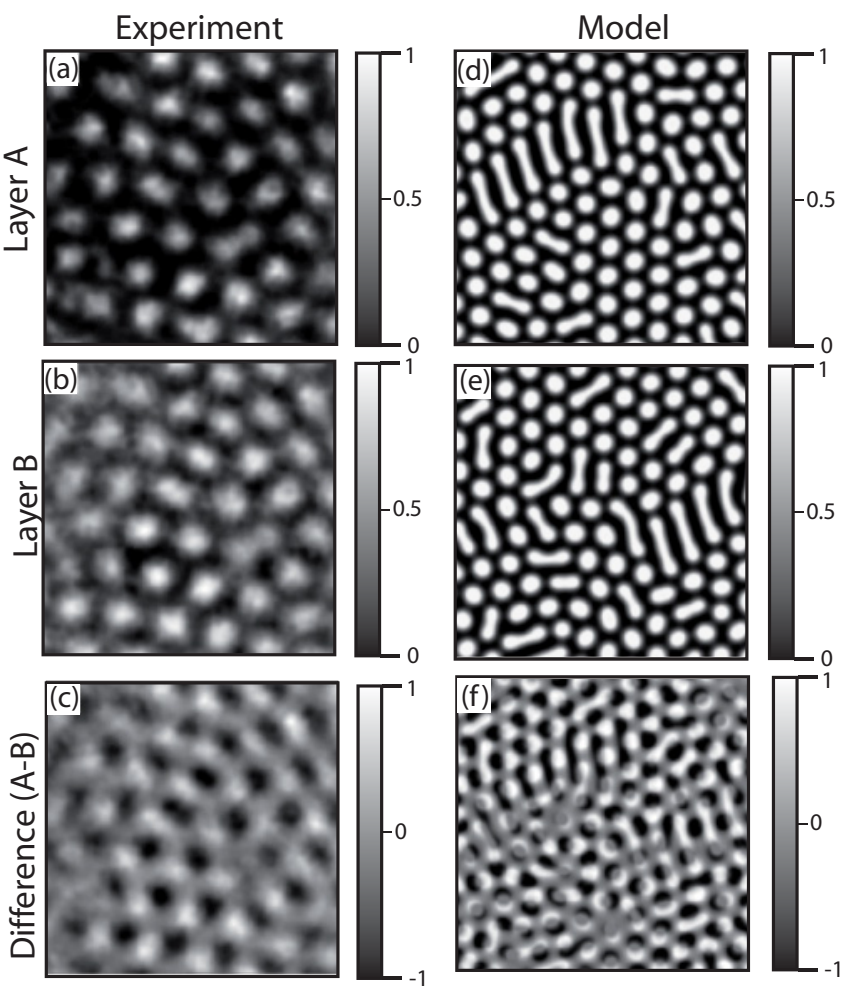

FIG. 2. Snapshots of the experiment of spots-spots interaction. (a) corresponds to the illuminated layer $A$ and (b) corresponds to layer $B$. (c) is constructed by subtracting (a) and (b) as described in the text. (d), (e), and (f) are the corresponding numerical simulations showing complete agreement with the experimental results. Size of the experimental pictures $=3.3 \times 3.3 \mathrm{~mm}$. Light intensity $=$ $500 \times 10^{-6} \pm 50 \times 10^{-6} \mathrm{~W} / \mathrm{cm}^{2}$. Light intensity for the simulations: $\phi_{a}=\phi_{b}=0$.

$$
\begin{aligned}
& \frac{\partial u_{b}}{\partial t}=a-c u_{b}-\frac{4 u_{b} v_{b}}{1+u_{b}^{2}}-\phi_{b}+\nabla^{2} u_{b}+k_{u}\left(u_{a}-u_{b}\right), \\
& \frac{\partial v_{b}}{\partial t}=\sigma\left(c u_{b}-\frac{u_{b} v_{b}}{1+u_{b}^{2}}+\phi_{b}+d \nabla^{2} v_{b}+k_{v}\left(v_{a}-v_{b}\right)\right) .
\end{aligned}
$$

The first set of equations describes the behavior in layer $A$ while the second set is for layer $B . u_{a}$ and $v_{a}$ are the dimensionless concentrations of activator and inhibitor species in layer $A$, while $u_{b}$ and $v_{b}$ corresponds to layer $B$. $a, c$, and $\sigma$ are dimensionless parameters related to other initial concentrations and rate constants, and $d$ is proportional to the ratio of diffusion coefficients between activator and inhibitor. $\phi_{a}$ and $\phi_{b}$ account for the rate of the photochemical reactions in layers $A$ and $B$, respectively. $k_{u}$ and $k_{v}$ represent the coupling strength among layers, and it is related to the difficulty of the chemicals to diffuse from one layer to the other through the membrane (these parameters can be experimentally controlled by selecting the type and number of membranes in between the two gel layers).

Parameters for the numerical simulations were selected to mimic the experimental results: $a=16, c=0,4, \sigma=20$, $d=1,07$. Under these circumstances and with no illumination $\left(\phi_{a}=\phi_{b}=0\right)$, the structure in both layers is composed of spots in hexagonal configuration [Figs. 2(d) and 2(e)].
The coupling is performed in such a way that allows all chemicals to diffuse between the layers. We consider a symmetric diffusion coefficient among the layers to be the same for both species and equal to $k_{u}=k_{v}=0.001$.

\section{RESULTS}

Different experiments were performed by varying the intensity of light in layer $A$ to explore the interaction between different Turing pattern configurations. The results of the experiments and the simulations suggest a complicated and unexpected interaction, which appears to be strongly dependent on the spatial configuration of the interacting patterns.

\section{A. Spots-spots interaction}

When the difference between the homogeneous illumination in the layers is small, the chemical conditions are similar in both systems. This way, we can study the interaction of two coupled hexagonal patterns. Figure 2(a) corresponds to the pattern in the illuminated layer $A$, and Fig. 2(b) is the pattern in the nonilluminated layer $B$. In both systems, the configuration is composed of spots in hexagonal ordering because the light intensity is not enough to influence the pattern configuration.

Correlation between the pattern in both layers is very high. Some mismatch between the structures can be observed, presumably due to the weakness of the diffusive coupling.

To compare the configuration of the patterns in both layers, we plot in Fig. 2(c) the results of the pixel-to-pixel subtraction of the intensity levels of the illuminated layer $A$ minus the intensity levels in the nonilluminated layer $B$. Figure 2(c) shows that the diffusive coupling rearranges the regions with high activator (dark color) in layer $A$ with regions of high inhibitor concentration (light color) in layer $B$. This way, the "holes" between spots in layer $A$ are occupied by the spots in layer $B$, and vice versa, and the patterns appear as if they were shifted spatially of a wavelength between the two layers. The same result can be observed in the numerical simulations shown in Figs. 2(d)-2(f).

\section{B. Stripes-spots interaction}

Slightly increasing the intensity of illumination shifts the chemical conditions of the illuminated layer toward a regime where stripes are the steady-state configuration of the pattern [see Fig. 3(a)], while the nonilluminated layer remained mostly composed of spots [see Fig. 3(b)]. This configuration allowed us to analyze the effect of the diffusive interaction between chemical patterns in different chemical conditions and spatial configurations.

When we analyze the difference between the two patterns [Fig. 3(c)], we observe that the spots in the nonilluminated layer $B$ occupied the space between the stripes of the illuminated layer $A$. Instead of forcing the nonilluminated pattern to shift its configuration to stripes, the spatial diffusive coupling between patterns of different spatial configurations acts in such a way that rearranges the two different patterns by occupying the locations of the low activator concentration in one layer by low inhibitor concentrations in the other. The numerical simulations [Figs. 3(d), 3(e), and the difference in 

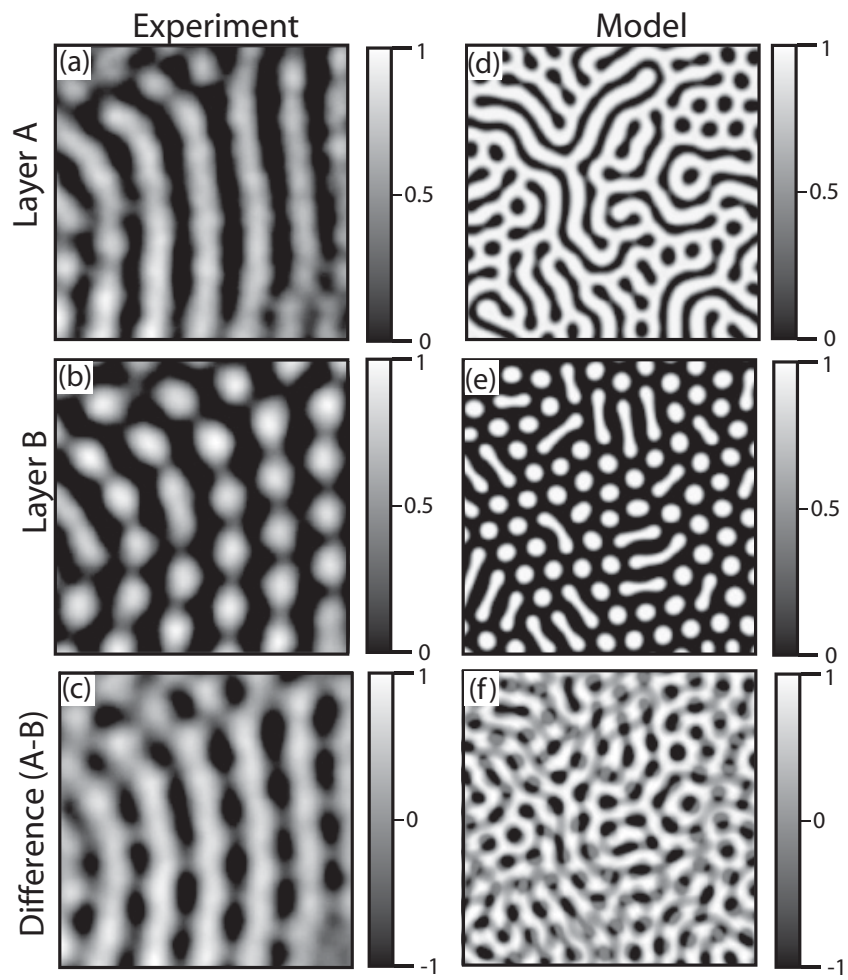

FIG. 3. Snapshots of the experiment of stripes-spots interaction. (a) corresponds to the illuminated layer $A$ and (b) corresponds to layer $B$. (c) is the difference between the configuration in layer $A$ and layer $B$. (d), (e), and (f) are the corresponding numerical simulations in agreement with experimental results. Size of each picture $=4.3 \times$ $4.3 \mathrm{~mm}$. Light intensity is $I=8500 \times 10^{-6} \pm 100 \times 10^{-6} \mathrm{~W} / \mathrm{cm}^{2}$. Light intensity for the simulations: $\phi_{a}=1.5 ; \phi_{b}=0$.

Fig. 3(f)] show the same arrangement of spots occupying the locations between the stripes of the illuminated pattern.

\section{Honeycomb-spots interaction}

Higher values of the light intensity yield to another possibility of interaction between different patterns. The pattern in layer $A$ is now composed of black spots in a hexagonal configuration, also called a "honeycomb-type" pattern. This configuration can be observed in Fig. 4(a), where only the upper left half of the pattern is illuminated with high light intensity of illumination (i.e., above the dashed line). In this case, we illuminate just half of the system to simultaneously observe the pattern under conditions of illumination (above the dashed line) an nonillumination (below the dashed line). In the other layer, the chemical conditions correspond to the spots regime, as we can still see in the area below the dashed line [see Fig. 4(b)]. This way, in the illuminated region, we induce a situation where there is mutual chemical interaction between antagonist pattern configurations, i.e., white spots (usually called $H_{0}$ ) in the nonilluminated layer with black spots $\left(H_{\pi}\right)$ or honeycomb pattern in the illuminated layer.

Surprisingly, instead of spots rearranging in the "holes" of the honeycomb pattern, the pattern in the noniluminated layer $B$ gets completely suppressed (above the dashed line, corresponding with the honeycomb pattern in the illuminated layer $A$ ). We hypothesize that in the situation where the
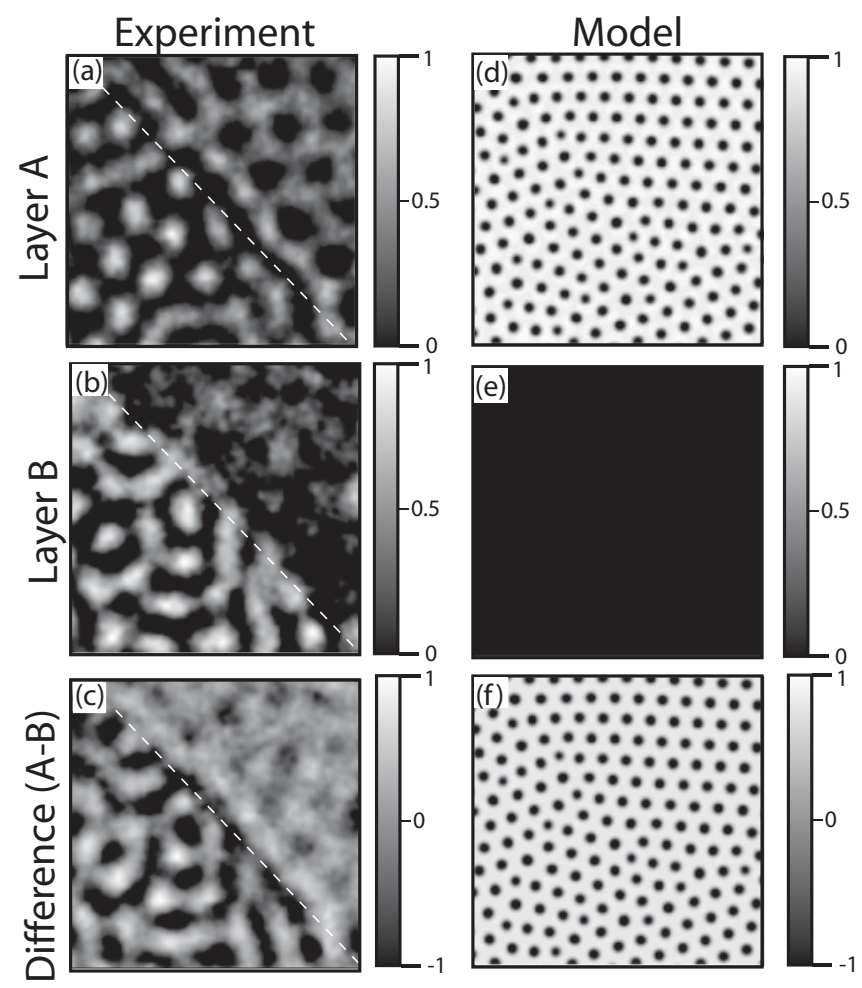

FIG. 4. Snapshots of the experiment of honeycomb-spots interaction. (a) corresponds to layer $A$, in which only the area below the dashed line is being illuminated. (b) corresponds to layer $B$. (c) is the difference between (a) and (b). (d), (e), and (f) are the corresponding numerical simulations in agreement with experimental results. Size of each picture $=4.3 \times 4.3 \mathrm{~mm}$. Light intensity $I=12700 \times 10^{-6} \pm 100 \times 10^{-6} \mathrm{~W} / \mathrm{cm}^{2}$. Light intensity for the simulations: $\phi_{a}=2.1 ; \phi_{b}=0$.

patterns are antagonistic (black spots coupled with white spots), the system is not able to properly reorganize for this chemical concentration and one of the patterns gets suppressed. Figure 4(c) is the difference between the pattern in the illuminated and nonilluminated layers. Again, the numerical simulations [Figs. 4(d), 4(e), and the difference in Fig. 4(f)] show the same behavior. It is important to note here that the light intensity that reaches the nonilluminated layer $B$ is unable to suppress the pattern by itself (the next section shows that higher intensities recover the pattern in the nonilluminated layer) so the annihilation of the pattern is only due to the interaction with the other Turing honeycomb layer.

\section{Steady state-spots interaction}

Finally, when the external light over the layer $A$ is sufficiently high, the pattern is suppressed (Fig. 5). This way, we can check the result of an interaction of an hexagonal pattern with a chemical steady state with no pattern. The result of this interaction is shown in Fig. 5. Interestingly, by increasing the illumination in the layer $A$, we allow recovery of the pattern in the nonilluminated layer, providing evidence that the honeycomb pattern was the cause of the annihilation of the nonilluminated spots due to the spatial interaction.

In layer $B$, the pattern is again clearly observed, and the main configuration is spots. Figure 5(c) shows the difference 


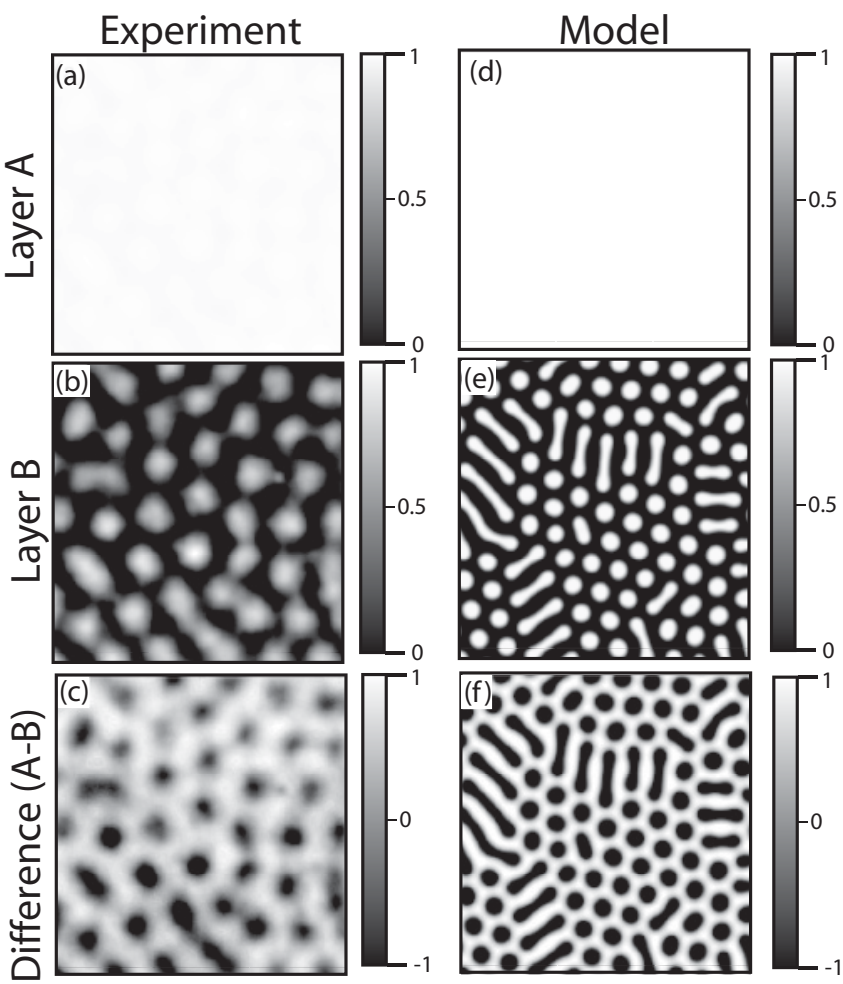

FIG. 5. Snapshots of the experiment of steady state-spots interaction. (a) corresponds to layer $A$, which is being illuminated with very high light intensity, and (b) corresponds to layer $B$. (c) is the difference between (a) and (b). (d), (e), and (f) correspond to numerical simulations in agreement with experimental results. Size of each picture $=4.2 \times 4.2 \mathrm{~mm}$. Light intensity $I=21200 \times 10^{-6} \pm 100 \times$ $10^{-6} \mathrm{~W} / \mathrm{cm}^{2}$. Light intensity for the simulations: $\phi_{a}=3 ; \phi_{b}=0$.

between the patterns in both layers, which in this case is just the pattern in layer $B$ inverted. Numerical simulations also report the same behavior under conditions of high light intensity [see Figs. 5(d)-5(f)].

\section{E. Numerical phase diagram}

In order to enlighten the different regimes observed in this system, we performed a detailed analysis of the different spatial arrangements that can be observed in both layers. The two control parameters considered are the coupling strength between layers, $k_{u}=k_{v}$, and the light intensity on layer $A$, $\phi_{a}$. This is shown in Fig. 6. Points in this figure correspond with different numerical simulations. Different symbols in the figure correspond to different spatial configurations and the meaning of the symbols is detailed in the figure caption.

All numerical simulations inside the shadowed area correspond with situations where both layers exhibit the same pattern, i.e., the coupling is strong enough or the light gradient is too weak so that both layers show intercalated patterns in a similar spatial configuration. For small values of the coupling or large light gradients, different patterns on both layers can be observed (outside the gray region). The dashed line separates regions where the illuminated layer $A$ exhibits a pattern (left to that line) from the range of parameters where the pattern is annihilated.

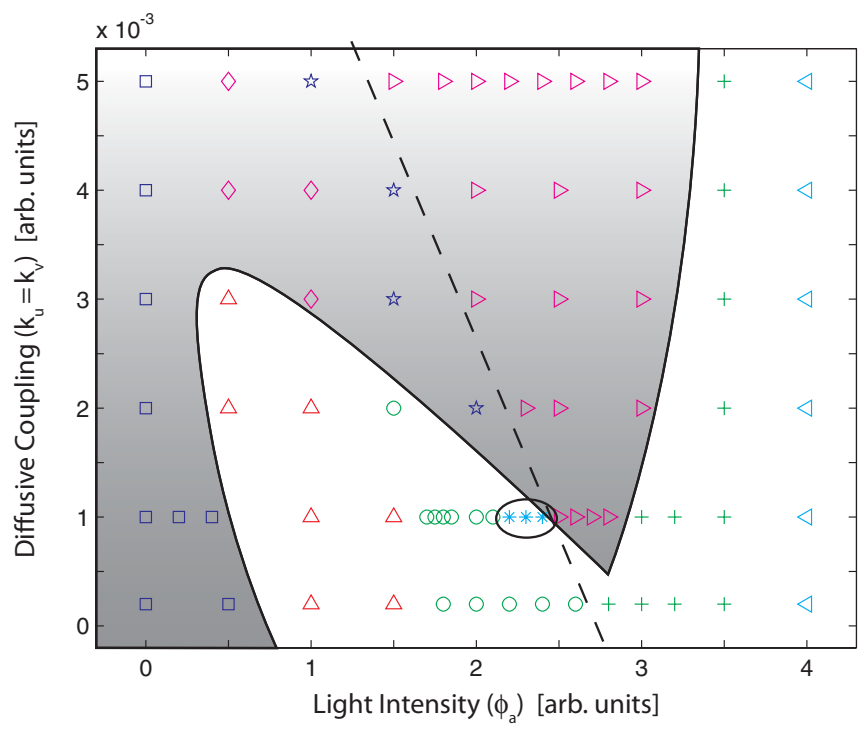

FIG. 6. (Color online) Numerical phase diagram showing the different behaviors observed for the relevant parameters in the system (namely, coupling strength $k_{u}=k_{v}$ and light intensity on layer $\left.A, \phi_{a}\right)$. Different symbols in the figure correspond with different spatial arrangements in both layers as follows: blue squares stand for hexagons $H_{0}$-hexagons $H_{0}$, red upward-pointing triangles stand for stripes-hexagons $H_{0}$, green circles stand for hexagons $H_{\pi}$-hexagons $H_{0}$, light blue stars stand for $H_{\pi}$-steady state, pink right-pointing triangles stand for steady state-steady state, green crosses stand for steady state-hexagons $H_{0}$, light blue left-pointing triangles stand for steady state-stripes, pink diamonds stand for stripes-stripes, and blue pentagrams stand for hexagons $H_{\pi}$-hexagons $H_{\pi}$ (where the first pattern corresponds to the illuminated layer $A$ and the second to the layer in darkness $B$ ). The gray area separates regions where the same type of pattern occurs in both layers from those regions where patterns are different in both layers. The dashed line separates regions where the light intensity is high enough to suppress the pattern in the illuminated layer. All other model parameters are the same as in previous simulations.

\section{DISCUSSION AND CONCLUSIONS}

We have performed an experimental and numerical study of the interaction between two "two-dimensional" Turing patterns in layers coupled via diffusion, allowing us to directly study interactions between different spatial configurations. By reducing the strength of the coupling (achieved by placing permeable membranes between the layers), we can photochemically shift the chemical conditions at both sides of the permeable membranes, and maintain a different pattern arrangement in the two sides of our system: stripes, hexagons $H_{\pi}$, and hexagons $H_{0}$. The characteristics of our setup allowed us to unveil a rich scenario of interactions between the different spatial configurations of the Turing structures. The coupling can induce either pattern intercalation or pattern annihilation, depending on the spatial configurations of the two interacting patterns. The numerical phase diagram in Fig. 6 shows an interesting scenario with a rich variety of regimes, some of them not observed experimentally due to the difficulty of systematically changing the strength of spatial coupling. Interestingly, as a consequence of the coupling, the pattern in 
the illuminated layer $A$ gets annihilated at smaller values of the light intensity $\phi_{a}$ when the pattern is increased (the dashed line is not vertical). The regime where the pattern on both layers coincides (inside of gray region) strongly depends on the light intensity and the coupling. The shape of this regime strongly resembles other familiar resonant phenomena (such as Arnold tongues), suggesting that a resonance in space between the two layers is probably taking place. Note that for the numerical simulations, annihilation of the nonilluminated layer while the illuminated layer still exhibits patterning is relatively small in the parameter space (points marked by light blue stars and encircled). On the contrary, the same phenomena can be observed experimentally in a robust way (honeycomb-spots interaction), providing evidence of a mismatch between the experimental system and the model.
In conclusion, the interaction between patterns has been an important subject of study in nonlinear dynamics; we believe our findings raise new questions in the understanding of how nonlinear spatial systems interact.

\section{ACKNOWLEDGMENTS}

This work has been supported by the DGI (Spain) under Project No. FIS2010-21023 and Xunta de Galicia (Spain) under Project Nos. PGIDIT05PXIC20607PN and INCITE07PXI206131ES and by the NSF (USA). D.G.M. acknowledges a Ramon y Cajal Fellowship from the Ministry of Science and Technology of Spain and a Marie Curie International Reintegration Grant from the EU 248346-NMSSBLS, as well as financial support from the CSIC-SPAIN (JAE-DOC).
[1] A. Pikovsky, M. Rosenblum, and J. Kurths, Synchronization: A Universal Concept in Nonlinear Sciences (Cambridge University Press, Cambridge, 2003).

[2] S. Strogatz, Sync: The Emerging Science of Spontaneous Order (Hyperion, New York, 2003).

[3] S. C. Manrubia, A. S. Mikhailov, and D. H. Zanette, Emergence of Dynamical Order: Synchronization Phenomena in Complex Systems (World Scientific, Singapore, 2004).

[4] S. J. Russell and P. Norvig, Artificial Intelligence: A Modern Approach (Pearson Education, NJ, 2003).

[5] J. Awrejcewicz, Bifurcation and Chaos in Coupled Oscillators (World Scientific, Singapore, 1991).

[6] L. Yang, M. Dolnik, A. M. Zhabotinsky, and I. R. Epstein, Phys. Rev. Lett. 88, 208303 (2002).

[7] L. Yang and I. R. Epstein, Phys. Rev. Lett. 90, 178303 (2003).

[8] Igal Berenstein, Milos Dolnik, Lingfa Yang, Anatol M. Zhabotinsky, and Irving R. Epstein, Phys. Rev. E 70, 046219 (2004).

[9] L. Yang and I. R. Epstein, Phys. Rev. E 69, 026211 (2004).

[10] I. Lengyel, G. Rábai, and I. R. Epstein, J. Am. Chem. Soc. 112, 4606 (1990).
[11] I. Lengyel, G. Rábai, and I. R. Epstein, J. Am. Chem. Soc. 112, 9104 (1990).

[12] A. M. Turing, Philos. Trans. R. Soc. B 237, 37 (1952).

[13] V. Castets, E. Dulos, J. Boissonade, and P. De Kepper, Phys. Rev. Lett. 64, 2953 (1990).

[14] A. P. Muñuzuri, M. Dolnik, A. M. Zhabotinsky, and I. R. Epstein, J. Am. Chem. Soc. 121, 8065 (1999).

[15] A. K. Horváth, M. Dolnik, A. P. Muñuzuri, A. M. Zhabotinsky, and I. R. Epstein, Phys. Rev. Lett. 83, 2950 (1999).

[16] S. Rüdiger, D. G. Míguez, A. P. Muñuzuri, F. Sagués, and J. Casademunt, Phys. Rev. Lett. 90, 128301 (2003).

[17] B. Peña, C. Pérez-García, A. Sanz-Anchelergues, D. G. Míguez, and A. P. Munuzuri, Phys. Rev. E 68, 056206 (2003).

[18] D. G. Míguez, E. M. Nicola, A. P. Muñuzuri, J. Casademunt, F. Sagués, and L. Kramer, Phys. Rev. Lett. 93, 048303 (2004).

[19] M. Dolnik, I. Berenstein, A. M. Zhabotinsky, and I. R. Epstein, Phys. Rev. Lett. 87, 238301 (2001).

[20] D. G. Míguez, Ph.D. thesis, Universidade de Santiago de Compostela, Spain, 2005.

[21] Q. Ouyang and Harry L. Swinney, Chaos 1, 411 (1991).

[22] Chemical Waves and Patterns, edited by R. Kapral and K. Showalter (Kluwer Academic, Dordrecht, 1995). 